\title{
Predictors of self-perceived health worsening over COVID-19 emergency in ALS
}

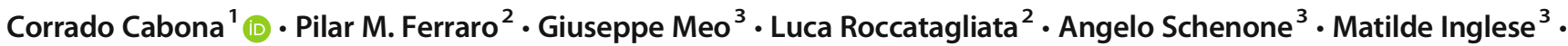 \\ Flavio Villani ${ }^{1} \cdot$ Claudia Caponnetto $^{3}$
}

Received: 30 September 2020 / Accepted: 12 December 2020 / Published online: 14 January 2021

(C) Fondazione Società Italiana di Neurologia 2021

\begin{abstract}
Introduction Factors influencing self-perceived health status over Corona Virus Disease 2019 (COVID-19) emergency in vulnerable populations, such as patients with chronic neurological diseases, are still unknown. In this work, we aimed at testing whether clinical care changes imposed by the quarantine, together with certain demographic and disease-specific features, might have determined a self-perceived worsening of health status in patients with amyotrophic lateral sclerosis (ALS).

Methods A brief web-based questionnaire investigating self-perceived anxiety, depression, and motor worsening, as well as clinical care changes over COVID-19 emergency, was administered to ALS patients currently followed at San Martino Hospital. Ordinal and logistic regression analyses were applied to identify significant predictors of health status.

Results Fifty-seven ALS patients completed the questionnaire. A total of $35.08 \%$ of cases reported anxiety symptoms, $36.84 \%$ depressive symptoms, and $35.08 \%$ reported worsening of motor symptoms. Significant predictors of anxiety symptoms severity included female gender, greater motor impairment, more aggressive disease course, and rehabilitation therapy suspension. The only significant predictor of depressive symptoms severity was a more aggressive disease course. Significant predictors of motor worsening were shorter disease duration and exams/visits cancelation.

Discussion COVID-19 emergency and its management exerted a significant impact on self-perceived health status in patients with ALS, particularly in those cases in the earliest disease phases and with a more aggressive disease course. These findings have potential to improve personalized medicine strategies in the next phase.
\end{abstract}

Keywords Corona virus disease $2019 \cdot$ Amyotrophic lateral sclerosis $\cdot$ Anxiety $\cdot$ Depression $\cdot$ Motor worsening

\section{Introduction}

The international outbreak of Corona Virus Disease 2019 (COVID-19) has caused a health system emergency worldwide, calling for restrictive measures to prevent further spread.

Corrado Cabona

corrado.cabona@gmail.com

1 Division of Clinical Neurophysiology and Epilepsy Center, Ospedale Policlinico San Martino, IRCCS, Largo R. Benzi 10,

16132 Genoa, Italy

2 Department of Neuroradiology, Ospedale Policlinico San Martino, IRCCS, Genoa, Italy

3 Department of Neurology, Ospedale Policlinico San Martino, IRCCS, Genoa, Italy
On 9 March 2020, the Italian government has imposed a national quarantine, suspending the majority of activities and mandating self-isolation.

While COVID-19 represents a severe health threat for everyone, many neurological patients are at increased risk compared to the general population [1]. Among them, patients with respiratory insufficiency from neuromuscular weakness, such as patients with Amyotrophic Lateral Sclerosis (ALS), are likely to be at higher risk of severe COVID-19 infection [2].

Besides a greater vulnerability in case of infection, ALS patients are also more vulnerable to the indirect effects of a quarantine since they are highly dependent on other figures (e.g., caregivers, neurologists, physical, and therapists) to manage their chronic disease and maintain a good health status.

Despite these considerations, COVID-19 emergency is so recent that clear data on its effects in patients with ALS are still missing. 
In this work, we aimed at testing whether clinical care changes imposed by the quarantine, together with certain demographic and disease-specific features, might have determined a self-perceived worsening of mental and physical health status in patients with ALS.

\section{Methods}

\section{Setting and participants}

Between March and April 2020, all the ALS patients currently followed at San Martino Hospital were invited to complete a web-based questionnaire.

Inclusion criteria for ALS patients are as follows: (1) a diagnosis of possible, probable or definite ALS according to current criteria [3]; (2) a detailed clinical characterization including time of symptoms onset, site of symptoms onset (bulbar or spinal), and clinical phenotype defined using current criteria $[4,5]$; (3) a clinical evaluation within 6 months comprehensive of the ALS functional rating scale revised (ALSFRS-r) [6], and the clinical progression rate estimation (calculated with the formula (48-ALFRS-r score at the time of the evaluation)/disease duration)); and (4) absence of cognitive impairment or overt frontotemporal dementia based on cognitive assessment at the time of the clinical evaluation.

\section{The questionnaire}

We developed a brief questionnaire specifically designed to investigate self-perceived mental and physical health status, as well as clinical care changes, over COVID-19 emergency.

\section{Self-perceived mental and physical health status}

Patients were asked to report the eventual presence of selfperceived anxiety and/or depressive symptoms over the quarantine period, and, if so, to rate them on a 3-point scale ranging from 1 "mild," to 2 "moderate," and 3 "severe."

Additionally, patients were asked to report the eventual presence of a self-perceived worsening of motor symptoms over the quarantine period, and, if so, to categorize it using the following categories: (1) selective motility decline, (2) selective respiratory worsening, (3) selective increase of pain symptoms, (4) a combination of motility and respiratory symptoms, (5) a combination of motility and pain symptoms, and (6) mixed symptoms (combined motility, respiratory, and pain symptoms).

\section{Clinical care changes}

ALS patients were asked to report if, over the quarantine period, they (1) experienced any difficulties in retrieving medicines, (2) had to cancel any exams and/or visits and, (3) in case they were following any rehabilitation therapy, if they had to suspend it over the quarantine period.

\section{Data analysis}

All data were analyzed using CRAN R Version 3.4.1. Demographic and disease-specific variables, as well as clinical care changes, were entered in regression models to identify significant predictors of self-perceived health-status. Ordinal regression analyses were applied to identify significant predictors of self-perceived anxiety and depressive symptoms severity, and logistic regression analyses were used to identify significant predictors of self-perceived motor worsening.

\section{Ethics approval and consent to participate}

The study was approved by the Institutional IRB, at the University of Genoa. Written informed consent was obtained from all participants (or guardians of participants) in the study.

\section{Results}

\section{Patients demographic and disease-specific features}

Out of the total sample of 71 ALS patients currently followed at San Martino Hospital and invited to participate, 57 cases $(40.36 \%$ females, $59.64 \%$ males) with a mean age of 66.54 years $(\mathrm{SD}=11.54)$ completed the questionnaire (Table 1).

Of ALS respondents, $17.54 \%$ had a bulbar onset ALS and $82.46 \%$ a spinal onset ALS (Table 1). A total of $52.63 \%$ of patients had a classic ALS phenotype, $12.28 \%$ a bulbar phenotype, $1.75 \%$ a flail arm phenotype, $8.78 \%$ a flail leg phenotype, $15.78 \%$ an UMN phenotype, and $8.78 \%$ a pseudopolyneuritic phenotype. The mean disease duration was 53.31 months $(\mathrm{SD}=35.08)$, the mean ALSFRS-r score was $30.59(\mathrm{SD}=11.36)$, and the mean clinical progression rate was $0.41(\mathrm{SD}=0.32)$. A total of $33.33 \%$ of patients were under treatment with noninvasive ventilation (NIV) (Table 1).

No statistically significant differences were observed between the sample of respondents and the sample of nonrespondents (Table 1).

\section{Self-perceived mental and physical health status}

A total of $35.08 \%$ of ALS patients reported anxiety symptoms, with a mean severity score of $1.65(\mathrm{SD}=0.74)$, and $36.84 \%$ reported depressive symptoms, with a mean severity score of $1.61(\mathrm{SD}=0.66)($ Table 2$)$.

Also, $35.08 \%$ of ALS patients reported a motor worsening. Among them, $50 \%$ experienced selective motility decline, 
Table 1 Demographic and disease-specific features of ALS patients

Total ALS sample $(N=71)$

\begin{tabular}{|c|c|c|c|}
\hline & Respondents & Non-respondents & $\begin{array}{l}P \\
\text { value }\end{array}$ \\
\hline Number & 57 & 14 & - \\
\hline Age [years] & $66.54(11.54)$ & $65.92(10.54)$ & 0.82 \\
\hline Gender [females] & $23(40.36 \%)$ & $7(50.00 \%)$ & 0.72 \\
\hline Site of onset [bulbar] & $10(17.54 \%)$ & $3(21.42 \%)$ & 1.00 \\
\hline $\begin{array}{l}\text { Clinical phenotype [classic/bulbar/flail arm/flail } \\
\text { leg/UMN/pseudopolyneuritic] }\end{array}$ & $\begin{array}{l}30(52.63 \%) / 7(12.28 \%) / 1(1.75 \%) / 5 \\
(8.78 \%) / 9(15.78 \%) / 5(8.78 \%)\end{array}$ & $\begin{array}{c}7(50.00 \%) / 2(14.28 \%) / 0(0.00 \%) / 1 \\
(7.14 \%) / 3(21.42 \%) / 1(7.14 \%)\end{array}$ & 0.98 \\
\hline Disease duration [months] & $53.31(35.08)$ & $50.57(41.03)$ & 0.10 \\
\hline ALSFRS-r [0-48] & $30.59(11.36)$ & $37.67(6.36)$ & 0.09 \\
\hline $\begin{array}{l}\text { Clinical progression rate [ALSFRS-r rate of decline } \\
\text { per month] }\end{array}$ & $0.41(0.32)$ & $0.44(0.47)$ & 0.60 \\
\hline Under treatment with NIV & $19(33.33 \%)$ & $4(28.57 \%)$ & 0.94 \\
\hline
\end{tabular}

Values are means (standard deviations) or frequencies (percentages). $P$ values refer to Mann-Whitney U-Test or Chi-Square test

Abbreviations: $A L S$, amyotrophic lateral sclerosis; ALSFRS-r, ALS functional rating scale-revised; NIV, noninvasive ventilation; UMN, upper motor neuron

$30 \%$ a combination of motility and respiratory symptoms, $5 \%$ selective respiratory worsening, $5 \%$ selective increase of pain symptoms, $5 \%$ a combination of motility and pain symptoms, and $5 \%$ mixed symptoms (Table 2)

\section{Clinical care changes}

Over the quarantine period, only $3.50 \%$ of ALS patients reported problems in retrieving medicines, while $63.15 \%$ of

Table 2 Self-perceived mental and physical health status and clinical care variables

Mental and physical health status

\begin{tabular}{ll}
\hline Self-perceived anxiety symptoms & $20(35.08 \%)$ \\
\hline Severity of self-perceived anxiety symptoms [1-3] & $1.65(0.74)$ \\
Self-perceived depressive symptoms & $21(36.84 \%)$ \\
Severity of self-perceived depressive symptoms [1-3] & $1.61(0.66)$ \\
Self-perceived clinical worsening & $20(35.08 \%)$ \\
Selective motility decline & $10(50.00 \%)$ \\
Motility and respiratory symptoms & $6(30.00 \%)$ \\
Selective respiratory worsening & $1(5.00 \%)$ \\
Selective increase of pain symptoms & $1(5.00 \%)$ \\
Motility and pain symptoms & $1(5.00 \%)$ \\
Mixed symptoms & $1(5.00 \%)$ \\
Clinical care & \\
Difficulties in retrieving medicines & $2(3.50 \%)$ \\
Exams and/or visits cancelation & $36(63.15 \%)$ \\
Following a rehabilitation therapy & $43(75.44 \%)$ \\
Rehabilitation therapy suspension & $34(79.06 \%)$
\end{tabular}

Values are means (standard deviations) or frequencies (percentages) patients had to cancel exams and/or visits (Table 2). A total of $75.44 \%$ of respondents were following a rehabilitation therapy at the time of the outbreak, and among these, $79.06 \%$ had to suspend it over the quarantine period (Table 2).

\section{Predictors of self-perceived mental and physical health worsening}

Significant predictors of more severe anxiety symptoms over the quarantine period were female gender $(P=0.04)$, more severe motor impairment (lower ALSFRS-r score, $P=0.02$ ), more aggressive disease course (higher clinical progression rate, $P<0.001)$, and rehabilitation therapy suspension $(P=$ 0.02) (Table 3).

The only significant predictor of more severe depressive symptoms over the quarantine period was a more aggressive disease course (higher clinical progression rate, $P=0.05$ ), while demographic and clinical care variables did not show any significant association (Table 3 ).

Significant predictors of a self-perceived motor worsening over the quarantine period were: shorter disease duration $(P=0.05)$, and exams and/or visits cancelation $(P=0.005)($ Table 3$)$.

\section{Discussion}

To our knowledge, this is the first study investigating both self-perceived mental and physical health status over COVID-19 emergency in patients with ALS.

More than $30 \%$ of respondents from our survey reported either anxiety, depressive, or motor worsening symptoms over 
Table 3 Ordinal and logistic regression analyses results

Severity of anxiety symptoms

\begin{tabular}{|c|c|c|}
\hline Variable & OR (CI 95\%) & $P$ value \\
\hline Age & $1.00(0.98,1.02)$ & 0.46 \\
\hline Gender [female] & $1.62(1.02,2.59)$ & 0.04 \\
\hline Site of onset [bulbar] & $0.80(0.42,1.49)$ & 0.49 \\
\hline Disease duration & $0.99(0.99,1.00)$ & 0.48 \\
\hline ALSFRS-r & $0.97(0.95,0.99)$ & 0.02 \\
\hline Clinical progression rate & $3.26(1.69,6.30)$ & $<0.001$ \\
\hline NIV [yes] & $1.18(0.71,1.96)$ & 0.51 \\
\hline Exams and/or visits cancelation [yes] & $1.59(0.98,2.56)$ & 0.06 \\
\hline Rehabilitation suspension [yes] & $1.70(1.07,2.70)$ & 0.02 \\
\hline \multicolumn{3}{|l|}{ Severity of depressive symptoms } \\
\hline Variable & OR (CI 95\%) & $P$ value \\
\hline Age & $1.00(0.98,1.02)$ & 0.48 \\
\hline Gender [female] & $1.46(0.92,2.32)$ & 0.10 \\
\hline Site of onset [bulbar] & $0.88(0.48,1.63)$ & 0.70 \\
\hline Disease duration & $1.00(0.99,1.00)$ & 0.69 \\
\hline ALSFRS-r & $0.98(0.96,1.00)$ & 0.19 \\
\hline Clinical progression rate & $1.99(1.00,3.96)$ & 0.05 \\
\hline NIV [yes] & $1.12(0.68,1.84)$ & 0.64 \\
\hline Exams and/or visits cancelation [yes] & $1.51(0.95,2.41)$ & 0.08 \\
\hline Rehabilitation suspension [yes] & $1.05(0.65,1.68)$ & 0.82 \\
\hline \multicolumn{3}{|l|}{ Motor worsening } \\
\hline Variable & OR (CI 95\%) & $P$ value \\
\hline Age & $1.01(0.96,1.06)$ & 0.64 \\
\hline Gender [female] & $1.84(0.61,5.67)$ & 0.27 \\
\hline Site of onset [bulbar] & $0.75(0.14,3.12)$ & 0.71 \\
\hline Disease duration & $0.97(0.95,0.99)$ & 0.05 \\
\hline ALSFRS-r & $1.00(0.95,1.05)$ & 0.94 \\
\hline Clinical progression rate & $4.49(0.83,32.62)$ & 0.09 \\
\hline NIV [yes] & $1.71(0.53,5.48)$ & 0.35 \\
\hline Exams and/or visits cancelation [yes] & $9.49(2.30,65.31)$ & 0.005 \\
\hline Rehabilitation suspension [yes] & $2.84(0.89,10.22)$ & 0.08 \\
\hline
\end{tabular}

Abbreviations: $O R$, odds ratio; $C I$, confidence interval

the quarantine period, suggesting a discrete impact of the emergency on this vulnerable population.

Concerning the mental health status, our findings are in line with recent studies describing an increase of anxiety and depression symptoms over the emergency compared to the preCOVID phase [7].

Notably, we further found that the presence and degree of a self-perceived health worsening was modulated by several factors, including demographic and disease-specific features, as well as clinical care changes imposed by the quarantine.

Significant predictors of more severe self-perceived anxiety symptoms over the quarantine period were female gender, more severe motor impairment, and a more aggressive disease course.
Significant associations between female gender and higher anxiety-depressive symptoms in ALS have already been reported in the literature [8], and more recently, a work by Ozdin and colleagues has identified female gender and accompanying chronic disease as significant risk factors for health anxiety during COVID-19 pandemic [9].

We observed that a faster disease progression was predictive of more severe anxiety symptoms. By definition, patients with fast disease progression rates are those with shorter disease duration and more severe motor impairment. Our results are therefore in agreement with recent studies reporting a significant decrease of anxiety over time [10] as well as higher levels of emotional well-being associated with slower disease progression in ALS [11].

Similarly, a more aggressive disease course further predicted more severe depressive symptoms, in line with previous studies reporting a decrease of depressive symptoms over time since diagnosis [12], and the well-known association between greater motor impairment and more severe depressive symptoms in ALS.

More than $30 \%$ of ALS patients in our cohort perceived a motor worsening over the quarantine period, with most common symptoms being selective motility decline, a combination of motility and respiratory symptoms, followed by pain and mixed symptoms.

To date, no study has investigated self-perceived motor worsening over the quarantine period in patients with ALS. However, the relative frequencies of symptoms reported in our sample are in agreement with the general manifestations of the disease, mainly mobility and respiratory deterioration, accompanied by variable degrees of pain symptoms [13].

Shorter disease duration was predictive of the presence of a self-perceived motor worsening, suggesting that patients in the earliest disease phases might be those more sensitive to subtle variations in their functional status.

We found that the vast majority of patients were subjected to clinical care changes over the quarantine period, mainly rehabilitation therapy suspension, and exams and/or visits cancelation. This finding is in line with recent studies in other affected countries, such as the USA, reporting COVID-19related challenges to ALS clinical care and research [14].

Notably, in line with recent reports describing a significant association between healthcare system changes during COVID19 emergency and anxiety in patients with ALS [15], we found that clinical care changes imposed by the quarantine exerted a significant impact on self-perceived anxiety in our sample.

Furthermore, we observed that these changes were significant predictors of a self-perceived motor worsening, highlighting the importance of continuous and integrated care management for self-perceived health status in neurological patients.

In this context, it is noteworthy that significant efforts have been made in order to handle ALS patients care management over COVID-19 emergency. The application of telemedicine, 
in particular, holds promise to enable maintaining a multidisciplinary approach to the care of patients [16] and monitoring disease progression [17] over the pandemic. A previous Italian study has shown that, even if the majority of patients and caregivers would have preferred a face-to-face visit, there was a global satisfaction with the telemedicine service [18]. A subsequent study has further confirmed these findings, showing that the majority of subjects were willing to continue the remote evaluation programs [19]. Taken together, these preliminary evidences suggest that telemedicine might be a valid tool to improve clinical management of ALS patients over the emergency.

The study is not without limitations. The first shortcoming deals with the relatively small ALS sample size, which was mainly determined by (a) stringent inclusion criteria (patients were included only if they had a clinical evaluation within 6 months and absence of cognitive impairment), and (b) the web-based administration of the questionnaire imposed by the quarantine, which limited the applicability in older patients. Furthermore, even if the absence of significant differences between the respondents and non-respondents samples in our study points towards a moderate generalizability of the results, the long mean disease duration and mild mean progression rate we observed in our cohort suggest the possibility that some of the newly diagnosed cases with short disease duration and rapid disease course might have been missed. Future studies in larger samples are therefore warranted to further explore the effects of COVID-19 emergency on this particularly vulnerable category of patients.

The second shortcoming is the absence of standardized anxiety and depression scales in order to avoid an overlap with the mood disorders scales recently administered as part of the routine behavioral examinations preceding the emergency.

Despite these limitations, our work provides a preliminary panoramic of the effects of COVID-19-related quarantine on self-perceived health status in patients with ALS.

Our results indicate that clinical care changes imposed by the emergency exerted significant negative impacts on patients with ALS, particularly in those in the earliest disease phases, and with a more aggressive disease course.

Knowledge of the factors contributing to self-perceived health worsening over the quarantine period in vulnerable populations, such as patients with ALS, is pivotal to better inform healthcare policies on adequate future strategies. These findings have potential to facilitate personalized medicine strategies in the next phase.

Acknowledgments The authors thank patients and their families for participating in the study.

Authors' contributions All authors contributed to the study conception and design. Material preparation, data collection, and analysis were performed by Corrado Cabona and Pilar Maria Ferraro. The first draft of the manuscript was written by Corrado Cabona and Pilar Maria Ferraro and all authors commented on previous versions of the manuscript. All authors read and approved the final manuscript.

Data availability Raw data are available upon appropriate request.

\section{Compliance with ethical standards}

Conflicts of interest The authors declare that they have no conflict of interest.

Ethical publication statement We confirm that we have read the Journal's position on issues involved in ethical publication and affirm that this report is consistent with those guidelines.

Code availability Not applicable.

\section{References}

1. Onder G, Rezza G, Brusaferro S (2020) Case-fatality rate and characteristics of patients dying in relation to COVID-19 in Italy. JAMA 323:1775-1776

2. Manji H, Carr AS, Browlee WJ, Lunn MP (2020) Neurology in the time of covid-19. J Neurol Neurosurg Psychiatry 91:568-570

3. Brooks BR, Miller RG, Swash M, Munsat TL (2000) World Federation of Neurology Research Group on Motor Neuron Diseases. El Escorial revisited: revised criteria for the diagnosis of amyotrophic lateral sclerosis. Amyotroph Lateral Scler Other Motor Neuron Disord 1(5):293-299

4. Chiò A, Calvo A, Moglia C, Mazzini L, Mora G, PARALS study group (2011) Phenotypic heterogeneity of amyotrophic lateral sclerosis: a population based study. J Neurol Neurosurg Psychiatry 82(7):740-746

5. Wijesekera LC, Mathers S, Talman P, Galtrey C, Parkinson MH, Ganesalingam J, Willey E, Ampong MA, Ellis CM, Shaw CE, alChalabi A, Leigh PN (2009) Natural history and clinical features of the flail arm and flail leg ALS variants. Neurology 72(12):10871094

6. Cedarbaum JM, Stambler N, Malta E, Fuller C, Hilt D, Thurmond $\mathrm{B}$ et al (1999) The ALSFRS-R: a revised ALS functional rating scale that incorporates assessments of respiratory function. BDNF ALS Study Group (Phase III). J Neurol Sci 169(1-2):13-21

7. De Lucia N, Ausiello FP, Spisto M, Manganelli F, Salvatore E, Dubbioso R (2020) The emotional impact of COVID-19 outbreak in amyotrophic lateral sclerosis patients: evaluation of depression, anxiety and interoceptive awareness. Neurol Sci 41(9):2339-2341

8. Cui F, Zhu W, Zhou Z, Ren Y, Li Y, Li M, Huo Y, Huang X (2015) Frequency and risk factor analysis of cognitive and anxietydepressive disorders in patients with amyotrophic lateral sclerosis/ motor neuron disease. Neuropsychiatr Dis Treat 11:2847-2854

9. Özdin S, Bayrak Özdin Ș (2020) Levels and predictors of anxiety, depression and health anxiety during COVID-19 pandemic in Turkish society: the importance of gender. Int J Soc Psychiat 66(5):504-511

10. Jakobsson Larsson B, Ozanne AG, Nordin K, Nygren I (2017) A prospective study of quality of life in amyotrophic lateral sclerosis patients. Acta Neurol Scand 136(6):631-638

11. Prell T, Steinbach R, Witte OW, Grosskreutz J (2019) Poor emotional well-being is associated with rapid progression in amyotrophic lateral sclerosis. eNeurologicalSci 16:100

12. Hillemacher T, Grässel E, Tigges S, Bleich S, Neundörfer B, Kornhuber J et al (2004) Depression and bulbar involvement in 
amyotrophic lateral sclerosis. Amyotroph Lateral Scler Other Motor Neuron Disord 5(4):245-249

13. Edge R, Mills R, Tennant A, Diggle PJ, Young CA, TONiC study group (2020) Do pain, anxiety and depression influence quality of life for people with amyotrophic lateral sclerosis/motor neuron disease? A national study reconciling previous conflicting literature. J Neurol 267(3):607-615

14. Andrews JA, Berry JD, Baloh RH, Carberry N, Cudkowicz ME, Dedi B et al (2020) Amyotrophic lateral sclerosis care and research in the United States during the COVID-19 pandemic: challenges and opportunities. Muscle Nerve 62(2):1

15. Consonni M, Telesca A, Dalla Bella E, Bersano E, Lauria G (2020) Amyotrophic lateral sclerosis patients' and caregivers' distress and loneliness during COVID-19 lockdown. J Neurol 21:1-4

16. De Marchi F, Cantello R, Ambrosini S, Mazzini L, CANPALS Study Group (2020) Telemedicine and technological devices for amyotrophic lateral sclerosis in the era of COVID-19. Neurol Sci 41(6):1365-1367
17. Bombaci A, Abbadessa G, Trojsi F, Leocani L, Bonavita S, Lavorgna L, Digital Technologies, Web and Social Media Study Group of the Italian Society of Neurology (2020) Telemedicine for management of patients with amyotrophic lateral sclerosis through COVID-19 tail. Neurol Sci 6:1-5

18. Vasta R, Moglia C, D'Ovidio F, Di Pede F, De Mattei F, Cabras S et al (2020) Telemedicine for patients with amyotrophic lateral sclerosis during COVID-19 pandemic: an Italian ALS referral center experience. Amyotroph Lateral Scler Frontotemporal Degener $12: 1-4$

19. Capozzo R, Zoccolella S, Musio M, Barone R, Accogli M, Logroscino $\mathrm{G}$ (2020) Telemedicine is a useful tool to deliver care to patients with amyotrophic lateral sclerosis during COVID-19 pandemic: results from southern Italy. Amyotroph Lateral Scler Frontotemporal Degener 21(7-8):542-548

Publisher's note Springer Nature remains neutral with regard to jurisdictional claims in published maps and institutional affiliations. 